\title{
Characteristics and Behaviors Associated with Prevalent SARS-CoV-2 Infection
}

This article was published in the following Dove Press journal:

International Journal of General Medicine

\author{
Sidney Aung (D) \\ Eric Vittinghoff ${ }^{2}$ \\ Gregory Nah' \\ Noah D Peyser (D) \\ Mark J Pletcher ${ }^{2}$ \\ Jeffrey E Olgin' \\ Gregory M Marcus' \\ 'Division of Cardiology, University of \\ California, San Francisco, San Francisco, \\ CA, USA; ${ }^{2}$ Department of Epidemiology \\ and Biostatistics, University of California, \\ San Francisco, San Francisco, CA, USA
}

Correspondence: Gregory M Marcus University of California, San Francisco, 505 Parnassus Ave, MI I80B, San Francisco, CA, 94143, USA

Tel + I 4I5-476-3450

Fax + 4 15-476-6260

Email greg.marcus@ucsf.edu
Introduction: Severe acute respiratory syndrome coronavirus 2 (SARS-CoV-2), which causes coronavirus disease 2019 (Covid-19), has been a serious threat to global health. Previous work has focused primarily on hospitalized patients or on identifying risk factors for disease severity and mortality once the infection has taken place. We sought to leverage the ubiquity of smartphones and mobile applications to study risk factors for Covid-19 infection in a large, geographically heterogenous cohort.

Methods: We analyzed data obtained from the Covid-19 Citizen Science (CCS) Study, a worldwide, mobile application-based cohort. After employing forward selection to identify variables with $\mathrm{p}$ values $<0.1$, multivariable logistic regression models were utilized to identify independent risk factors associated with prevalent SARS-CoV-2 infection.

Results: Among 36,041 participants in 113 countries and all 50 states in the US, 484 participants had prevalent SARS-CoV-2 infection. After multivariable adjustment, being a healthcare worker, living with at least one school-aged child, having pets at home, and having immunodeficiency were each associated with an increased odds of SARSCoV-2. The association between pets and prevalent SARS-CoV-2 was driven by dog ownership. After adjustment for the same covariates, Asian or Pacific Islander race, receiving a flu shot within the past year, increased level of education, and smoking or vaping marijuana within the last 30 days were each associated with a lower odds of SARS-CoV-2.

Conclusion: We identified various characteristics and behaviors, many of which are potentially modifiable, associated with prevalent SARS-CoV-2 infection in a world-wide mobile application-based cohort.

Keywords: COVID-19, epidemiology, cohort study, digital health, mobile applications

\section{Introduction}

Severe acute respiratory syndrome coronavirus 2 (SARS-CoV-2), which causes coronavirus disease 2019 (Covid-19), has had a significant impact on communities across the globe. Identifying risk factors associated with Covid-19 may prove valuable to the delivery of targeted public health interventions, providing more personalized advice to high-risk patient populations, and better informing the global response to the pandemic. Participation among community members and organizations has played a key role during the Covid-19 pandemic, ${ }^{1}$ and identifying potentially modifiable risk factors that can support community-based interventions is vital for containment of the pandemic. 
Previous work has focused on hospitalized patients ${ }^{2}$ or on disease severity and mortality once infection has taken place. ${ }^{3}$ Furthermore, smartphone technology on its own, and as an extension of telemedicine, has had various applications during the Covid-19 pandemic. $^{4}$ We therefore sought to leverage the ubiquity of smartphones and mobile applications to study risk factors for Covid-19 infection in a large, geographically heterogenous cohort.

\section{Methods}

We conducted a cross-sectional study using data obtained from the Covid-19 Citizen Science (CCS) Study, a worldwide, mobile application-based cohort (https:// covid19.eurekaplatform.org). Surveys pertinent to the current analyses are included in the Supplemental Materials. Questions where "effect-cause" relationships could obscure inferred causal relationships with prevalent disease were excluded.

Prevalent SARS-CoV-2 infection was defined as a history of a positive polymerase chain reaction (PCR) test for active infection. Participants were initially screened in the baseline survey for a history of any positive test for infection. Given an affirmative response to that initial question, participants were asked to distinguish between a PCR test for active infection versus an antibody test for prior infection. Self-report was verified by documentation of test results (Supplemental

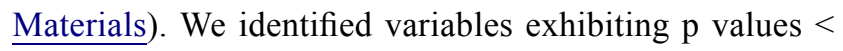
0.1 for associations with prevalent disease after adjusting for age, sex, race/ethnicity and then employed forward selection in a multivariable logistic regression model, retaining variables with $\mathrm{p}$ values $<0.1$. Statistical analyses were performed using Stata, version 16 (College Station, TX). Two-tailed p-values $<0.05$ were considered statistically significant. The study was approved by the University of California, San Francisco Institutional Review Board. All participants provided informed electronic consent.

\section{Results}

Among 36,041 participants in 113 countries and all 50 states in the US, 484 participants had prevalent SARSCoV-2 infection (Supplemental Figure 1) (4297 participants had a negative test, and 31,260 participants were not tested). Baseline participant characteristics are shown in Table 1. After multivariable adjustment, being a healthcare worker, living with at least one school-aged child, having pets at home, and having immunodeficiency were each associated with an increased odds of SARS-CoV-2 (Figure 1). The association between pets and prevalent SARS-CoV-2 was driven by dog ownership (Supplemental Tables 1 and 2). After multivariable adjustment for the same covariates, Asian or Pacific Islander race, receiving a flu shot within the past year, increased level of education, and smoking or vaping marijuana within the last 30 days were each associated with a lower odds of SARS-CoV-2 (Figure 1).

\section{Discussion}

We identified characteristics associated with prevalent SARS-CoV-2 infection in a world-wide mobile application-based cohort. Our observation that healthcare workers experience a higher likelihood of infection has biological plausibility and fits with previous evidence. ${ }^{5}$ Although disease transmission has been described from children ${ }^{6}$ and may theoretically occur from pets, ${ }^{7}$ these data suggest that each may be an independent risk factor for infection. A higher risk among dog owners may arise from environmental exposures during dog-walking or fomite-based exposure from dog petting.

Although previous evidence demonstrates a higher risk of infection among black and LatinX individuals, ${ }^{8}$ here we found that Asians experience the lowest risk. A higher degree of education is commonly a protective factor against disease. The observation that recent marijuana smoking or vaping was associated with a lower risk was surprising-while it is possible this reflected "effect-cause" (for example, those feeling ill were less likely to use the drug), we did not find a similar association with e-cigarettes or smoking tobacco. While the flu vaccine would not be considered to confer crossimmunity with coronaviruses, the lower risk among those who received the flu shot could reflect more overall healthy behaviors or perhaps reflects a general immune system boost that renders individuals less prone to viral infection.

While a strength of the current study is that it was not constrained to individuals seeking medical care, we acknowledge that we cannot exclude residual or unmeasured confounding in this observational study and that our study population, all with smartphones, does not 
Table I Baseline Characteristics of Participants with and without a Positive SARS-CoV-2 Test for Active Infection

\begin{tabular}{|c|c|c|c|}
\hline & $\begin{array}{l}\text { Tested Positive } \\
\mathbf{N}=\mathbf{4 8 4}\end{array}$ & $\begin{array}{l}\text { Everyone Else (Tested Negative, Not Tested) } \\
\mathrm{N}=35,557\end{array}$ & p-value \\
\hline Primary Residence in USA & $46 \mid(95.2 \%)$ & $32,989(92.8 \%)$ & 0.033 \\
\hline Median Age (IQR) & $42.0(36.0-51.0)^{\mathrm{a}}$ & $43.0(35.0-54.0)^{\mathrm{b}}$ & 0.17 \\
\hline Female Biological Sex & $36 \mid(75.8 \%)$ & $23,163(65.1 \%)$ & $<0.001$ \\
\hline Race/Ethnicity & & & $<0.001$ \\
\hline White & $403(84.7 \%)$ & $28,902(81.3 \%)$ & \\
\hline Black & $7(1.5 \%)$ & $336(0.9 \%)$ & \\
\hline Hispanic (any race) & 47 (9.9\%) & $2581(7.3 \%)$ & \\
\hline Asian or Pacific Islander & $10(2.1 \%)$ & $1919(5.4 \%)$ & \\
\hline Other (including multiracial) & $9(1.9 \%)$ & $813(2.3 \%)$ & \\
\hline Highest Level of Education & & & $<0.001$ \\
\hline Less than high school & $3(0.6 \%)$ & $164(0.5 \%)$ & \\
\hline High school graduate & $27(5.7 \%)$ & $1356(3.8 \%)$ & \\
\hline College Graduate (including associate degree) & $268(55.4 \%)$ & 17,552 (49.4\%) & \\
\hline Graduate school & $17 \mid(35.3 \%)$ & $15,035(42.3 \%)$ & \\
\hline Other & $7(1.4 \%)$ & $427(1.2 \%)$ & \\
\hline Healthcare worker & $181(37.4 \%)$ & 7299 (20.5\%) & $<0.001$ \\
\hline Children living with you & $205(42.4 \%)$ & $11,529(32.4 \%)$ & $<0.001$ \\
\hline Children home from college living with you & $40(8.3 \%)$ & $2778(7.8 \%)$ & 0.67 \\
\hline Any pets at home & $357(73.8 \%)$ & $22,882(64.4 \%)$ & $<0.001$ \\
\hline Cigarettes: any use in last $\mathbf{3 0}$ days & $25(5.4 \%)$ & $1906(5.4 \%)$ & 0.92 \\
\hline E-cigarettes: any use in last 30 days & $13(2.8 \%)$ & $1041(2.9 \%)$ & 0.89 \\
\hline Marijuana: any use in last 30 days & $31(6.8 \%)$ & $3300(9.3 \%)$ & 0.027 \\
\hline Atrial fibrillation & $7(1.5 \%)$ & $952(2.7 \%)$ & 0.09 \\
\hline Anemia & $54(11.4 \%)$ & $3512(9.9 \%)$ & 0.36 \\
\hline Asthma & $52(11.0 \%)$ & $3482(9.8 \%)$ & 0.49 \\
\hline Cancer & $14(3.0 \%)$ & $1023(2.9 \%)$ & 1.00 \\
\hline Coronary artery disease & $8(1.7 \%)$ & $825(2.2 \%)$ & 0.45 \\
\hline Congestive heart failure & $3(0.6 \%)$ & $220(0.6 \%)$ & 1.00 \\
\hline Chronic obstructive pulmonary disease & $10(2.1 \%)$ & $569(1.6 \%)$ & 0.46 \\
\hline Diabetes & $18(3.8 \%)$ & $1400(3.9 \%)$ & 0.91 \\
\hline High blood pressure & $86(18.1 \%)$ & 6777 (19.1\%) & 0.48 \\
\hline Human immunodeficiency virus & $4(0.8 \%)$ & $139(0.4 \%)$ & 0.13 \\
\hline Immunodeficiency & $20(4.2 \%)$ & $70 \mathrm{I}(2.0 . \%)$ & 0.003 \\
\hline Myocardial infarct & $3(0.6 \%)$ & $340(1.0 \%)$ & 0.64 \\
\hline Pregnant & $10(2.1 \%)$ & $306(0.9 \%)$ & 0.011 \\
\hline Sleep apnea & 45 (9.5\%) & $3578(10.1 \%)$ & 0.60 \\
\hline
\end{tabular}


Table I (Continued).

\begin{tabular}{|l|l|l|l|}
\hline & $\begin{array}{l}\text { Tested Positive } \\
\mathbf{N}=\mathbf{4 8 4}\end{array}$ & $\begin{array}{l}\text { Everyone Else (Tested Negative, Not Tested) } \\
\mathbf{N}=\mathbf{3 5 , 5 5 7}\end{array}$ & p-value \\
\hline Stroke & $5(1.1 \%)$ & $433(1.2 \%)$ & 1.00 \\
\hline Received flu shot within the year & $352(72.9 \%)$ & $26,258(73.8 \%)$ & 0.64 \\
\hline
\end{tabular}

Notes: ${ }^{a}$ The youngest and oldest ages of participants within the "Tested Positive" group was 18 years and 78 years, respectively. ${ }^{\mathrm{b}}$ The youngest and oldest ages of participants within the "Everyone Else" group was 18 years and 91 years, respectively.

necessarily reflect the general population. We also relied on interpretation from participants regarding what constituted a personal history of immunodeficiency, where immunodeficiency represents a broad phenomenon, though we included separate survey questions assessing a history of more specific immunosuppressive conditions such as for diabetes and human immunodeficiency virus (HIV). There is also the possibility that those who smoke or vape marijuana may have been less likely to receive SARS-CoV-2 testing, which could have ultimately contributed to the observed inverse relationship between smoking or vaping marijuana and prevalent SARS-CoV-2 infections. Furthermore, the analyses treated participants who were not tested for SARS-CoV-2 infection as if they had not been infected and we acknowledge that there may have been participants in this group with prevalent infections that were therefore under-ascertained. We do not believe this would have led to spurious false-positive results, but on the contrary, this may have led to underestimating the strengths of our observed relationships.

\section{Conclusion}

In conclusion, we identified various characteristics and behaviors, many of which are potentially modifiable, that are associated with prevalent SARS-CoV-2 infection in a world-wide mobile application-based cohort. We believe that these findings will be broadly useful for targeted

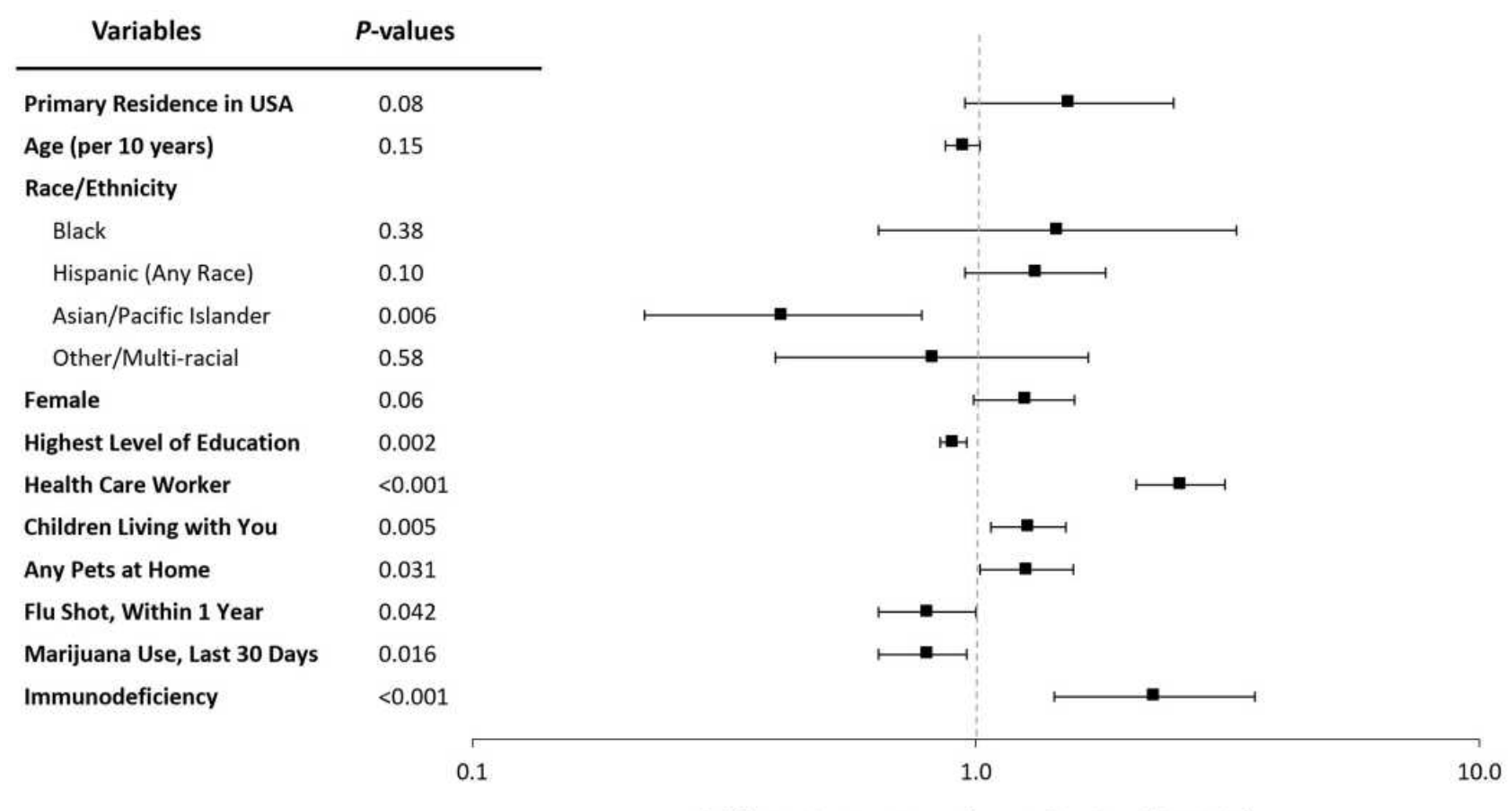

Odds Ratio For Prevalent Infection (95\% Cl)

Figure I Forest plot of adjusted odds ratios for participants with prevalent positive SARS-CoV-2 test results. Race/ethnicity categories were compared against non-Hispanic white participants. Y error bars indicate $95 \%$ confidence intervals. 
public health interventions in efforts to further contain the pandemic.

\section{Data Sharing Statement}

The data that support the findings of this study are available from the corresponding author upon reasonable request.

\section{Funding}

This study was funded by grants IU2CEB021881-01 and 3U2CEB021881-05S1 from the National Institute of Biomedical Imaging and Bioengineering at the National Institutes of Health to Drs. Marcus, Olgin, and Pletcher. The sponsors had no role in study design, data collection, analysis, interpretation of the data, writing of the paper, or the decision to publish the paper.

\section{Disclosure}

Dr Mark J Pletcher reports grants from PCORI, NIH, and Bill and Melinda Gates Foundation, during the conduct of the study. The authors report no other conflicts of interest in this work.

\section{References}

1. Sahu KK, Kumar R. Preventive and treatment strategies of COVID-19: from community to clinical trials. J Family Med Prim Care. 2020;9(5):2149-2157.

2. Richardson S, Hirsch JS, Narasimhan M, et al. Presenting characteristics, comorbidities, and outcomes among 5700 patients hospitalized with COVID-19 in the New York City area. JAMA. 2020;323 (20):2052-2059.

3. Cummings MJ, Baldwin MR, Abrams D, et al. Epidemiology, clinical course, and outcomes of critically ill adults with COVID-19 in New York City: a prospective cohort study. Lancet. 2020;395 (10239):1763-1770.

4. Iyengar K, Upadhyaya GK, Vaishya R, Jain V. COVID-19 and applications of smartphone technology in the current pandemic. Diabetes Metab Syndr. 2020;14(5):733-737.

5. Nguyen LH, Drew DA, Graham MS, et al. Risk of COVID-19 among front-line health-care workers and the general community: a prospective cohort study. Lancet Public Health. 2020;5(9):e475e483.

6. Park YJ, Choe YJ, Park O, et al. Contact tracing during coronavirus disease outbreak, South Korea, 2020. Emerg Infect Dis. 2020;26 (10):2465-2468.

7. Kiros M, Andualem H, Kiros T, et al. COVID-19 pandemic: current knowledge about the role of pets and other animals in disease transmission. Virol J. 2020;17(1):143.

8. Vahidy FS, Nicolas JC, Meeks JR, et al. Racial and ethnic disparities in SARS-CoV-2 pandemic: analysis of a COVID-19 observational registry for a diverse US metropolitan population. BMJ Open. 2020;10(8):e039849.

\section{Publish your work in this journal}

The International Journal of General Medicine is an international, peer-reviewed open-access journal that focuses on general and internal medicine, pathogenesis, epidemiology, diagnosis, monitoring and treatment protocols. The journal is characterized by the rapid reporting of reviews, original research and clinical studies across all disease areas. The manuscript management system is completely online and includes a very quick and fair peer-review system, which is all easy to use. Visit http://www.dovepress.com/ testimonials.php to read real quotes from published authors. 